\title{
Arbor
}

\section{Recursos Naturales}

\author{
Enrique Macpherson Mayol
}

Arbor CLXVI, 653 (Mayo 2000), 37-49 pp.

El Area se orienta hacia el fomento de la investigación de los procesos biológicos, geológicos, oceanográficos y, en general, medioambientales, que tienen lugar en la atmósfera, litosfera y biosfera. Esta investigación permite conocer los recursos y su evaluación, aumentar el conocimiento del mundo en que vivimos, además de contribuir al entendimiento y resolución de importantes problemas que afectan a la calidad de vida de la sociedad. La investigación sobre los recursos naturales es actualmente una prioridad fundamental en todos los países de nuestro entorno y se considera que el conocimiento de tales procesos es imprescindible para una correcta gestión de cualquiera de los recursos considerados. Este creciente valor puede permitir a nuestro país establecer una posición estratégica a medio y largo plazo sobre el uso de sus recursos naturales, así como una interacción necesaria e imprescindible a nivel científico y tecnológico para su consecución. El Area de Recursos Naturales es, por su naturaleza, pluridisciplinar por lo que se deben favorecer proyectos que permitan una interacción real entre diferentes grupos de científicos, con el fin de poder responder a preguntas concretas de nuestra sociedad. En la actualidad el Area está constituida por 18 Institutos, seis de ellos mixtos, aunque son 26 los Institutos que cuentan con departamentos que pertenecen al Area. En total trabajan en el Area algo más de 1.600 personas, incluyendo el personal universitario adscrito a los Centros Mixtos. Los Centros del Area se ubican en diversas comunidades: Andalucía, Aragón, Baleares, Cataluña, Canarias, Castilla-La Mancha, Castilla-León, Galicia, Madrid, Navarra y Valencia. 


\section{Definición}

Las investigaciones que se realizan en el Area de Recursos Naturales, por su compleja temática, presentan una gran diversidad disciplinaria. Se abordan dentro de ella investigaciones de muy diversa índole que, espacialmente, abarcan niveles locales, regionales y globales. En el Area existen grandes subáreas de investigación, encuadradas tanto en sistemas terrestres como marinos, y que reflejan la enorme hetereogeneidad de los estudios que realiza el CSIC en las Ciencias de la Naturaleza.

Los objetivos de los estudios que el CSIC realiza en este ámbito permiten aumentar nuestro conocimiento sobre el funcionamiento de la Tierra y posibilitan, no sólo resolver con más eficacia posibles efectos negativos de la actividad antrópica, sino también aprovechar las oportunidades que se deriven de esos cambios pudiendo, asimismo, desarrollar estrategias sostenibles para el aprovechamiento y uso óptimo de nuestro entorno.

El Area se orienta hacia el fomento de la investigación de los procesos biológicos, geológicos, oceanográficos y, en general, medioambientales, que tienen lugar en la atmósfera, litosfera y biosfera. Esta investigación permite conocer los recursos y su evaluación, aumentar el conocimiento del mundo en que vivimos, además de contribuir al entendimiento y resolución de importantes problemas que afectan a la calidad de vida de la sociedad. El objetivo fundamental es comprender el sistema Tierra, para proporcionar unas bases sólidas en que apoyar las actividades humanas presentes y futuras, tales como las actividades extractivas, la energía, la construcción de obras civiles, la gestión de residuos, la mejora de las condiciones de vida de la población, el uso de las aguas, la ordenación territorial, etc. Además, comprender los procesos que tienen lugar en nuestro entorno nos permitirá una mejor utilización de nuestros recursos, imprescindibles para el desarrollo del país y que representan un importante eslabón en la cadena industrial y en las necesidades sociales.

\section{Antecedentes}

En los inicios de la década de los setenta, la organización y ubicación de los Institutos que actualmente se encuadran en el Area de Recursos Naturales mostraban un panorama algo diferente del actual. No solo en lo referente a la calidad y cuantía de los equipamientos e insta- 


\section{Recursos Naturales}

laciones, sino también en cuanto a personal, internacionalización de las investigaciones e incidencia en la sociedad.

Los Institutos del Area se encontraban encuadrados en los antiguos Patronatos Alonso de Herrera y Juan de la Cierva, sufriendo la primera reorganización en 1971 que agrupaba al Instituto Nacional de Edafología y Agrobiología, Instituto de Botánica, Instituto Nacional de Geología, Estación Biológica de Doñana, Museo Nacional de Ciencias Naturales, Instituto de Investigaciones Pesqueras, Centro Pirenaico de Biología Experimental, Instituto Español de Entomología e Instituto de Parasitología. Algunos de estos Institutos estaban constituidos por varios centros, destancando el Nacional de Edafología con 21 centros.

Esta reorganización se vio, asimismo, modificada notablemente con el nuevo Reglamento de 1978, donde se separan las Areas de Ciencias Agrarias de las de Ciencias de la Tierra y del Espacio. En esta última se encuadrarían los Institutos que actualmente conforman el Area de Recursos Naturales, así como los Institutos de Astrofísica, que posteriormente pasarían al Area de Físicas.

En la actualidad el Area está constituida por 18 Institutos, cuatro de ellos mixtos y uno asociado, aunque son 26 los Institutos que cuentan con departamentos que pertenecen al Area. En total trabajan en el Area algo más de 1600 personas, incluyendo el personal universitario adscrito a los Centros Mixtos. Cabría destacar el elevado número de personal en formación o contratado, alrededor de 600 , contando con algo menos de 200 científicos de plantilla.

El aumento de la actividad investigadora en las últimas décadas, se ha visto favorecido por la adquisición de infraestructuras llamativas, como los buques oceanográficos García del Cid y Mytilus, que deben unirse al BIO Hespérides, y numerosos equipamientos de laboratorio y de campo, que han permitido realizar actividades científicas equiparables a las de cualquier país de nuestro entorno. Como reflejo de la calidad de estas actividades, el Area obtiene con notable éxito financiación en los Programas de la Unión Europea (superando el centenar de proyectos al año), así como en convocatorias nacionales y en contratos con empresas. Dicha actividad se traduce, por otra parte, en un claro aumento en el número y calidad de las publicaciones, superando en los últimos años las 5 cinco publicaciones por científico, de las cuales más de 2 corresponden a revistas SCI.

$\mathrm{El}$ incremento del interés social por las actividades relacionadas con el Medio Ambiente, hacen del Area uno de los focos de interés científico para el futuro, al que el CSIC no está siendo ajeno. Sirvan como ejemplo, la reciente constitución de nuevos Centros en Castilla 
La Mancha y Navarra, dirigidos hacia actividades cinegéticas y conservación de los recursos, respectivamente.

\section{Presente de la investigación en Recursos Naturales en el CSIC}

Los estudios sobre recursos naturales representan un campo de investigación de gran importancia en España y la potenciación de los mismos en los últimos años ha llevado a situar a nuestro país entre los doce primeros países productores de ciencia y tecnología ambiental del mundo. Los grupos de I+D españoles han logrado un excelente nivel y una participación activa en programas nacionales e internacionales, gracias a la incorporación de un número creciente de jóvenes investigadores así como la adquisición modernas infraestructuras de investigación.

El Area desarrolla una amplia gama de líneas de investigación, como lo demuestra la diversidad de Programas Nacionales donde los investigadores han obtenido en sus proyectos del último Plan Nacional de I+D: Medio Ambiente, Clima, Recursos Hídricos, Ciencias y Tecnologías Marinas, Antártida y Promoción General del Conocimiento. Una distribución similar se observa en los proyectos obtenidos en el IV Programa Marco de la UE.

En general, se han desarrollado proyectos en todas las líneas de investigación incluidas en el Area, aunque con ciertas diferencias en cuanto a éxito en las convocatorias. Estas diferencias están algo más asociadas a algunos Institutos y grupos, que a la propia naturaleza de las líneas. No obstante, se observa un incremento global generalizado en la obtención de proyectos nacionales, UE y contratos en el último cuatrienio, respecto al anterior. Una tendencia similar se observa en el número de publicaciones. Esta dirección hacia una mejora en la calidad de la investigación desarrollada en el Area se refleja también en que, alrededor de la mitad de las publicaciones incluidas en el SCI se encuentran en revistas de la mitad superior del ranking dentro de sus respectivos ámbitos. La capacidad de transferir resultados a la industria (medida como número de patentes) es, sin embargo, baja.

En general, el tamaño de los grupos en proyectos del Plan Nacional es mediano, siendo 3-4 investigadores por proyecto el equipo más común. Hay una escasa participación de investigadores de diferentes centros en un mismo equipo.

Las fuentes de financiación del Area son principalmente los Planes Nacionales y el IV Programa UE, con una tasa media de autofinanciación de alrededor del 29\%. Por líneas de investigación destacan las ciencias 
marinas y estructura y dinámica de la litosfera, con Institutos que superan el $35 \%$.

La cantera, entendida como número de investigadores contratados, es de unos 70-80 doctores. Esta cifra oscila según los años y, sobre todo, si se tienen en cuenta los doctores contratados con cargo a proyectos UE. Las líneas con una cantera más numerosa (mínimo de 4) son : Geoquímica ambiental, Evolución y Dinámica de la litosfera, Estructura y Procesos oceánicos, Sistemas litorales, Ecología evolutiva y del comportamiento, Ecología de poblaciones y Fauna Ibérica. Otras líneas tienen doctores de notable calidad, pero generalmente con un número inferior. El número de Tésis doctorales dirigidas en todo el Area se situa entre 50 y 60 por año.

En general, en todas las líneas de investigación hay grupos de buena calidad, que obtienen proyectos de una forma competitiva en las diferentes convocatorias. La mayor parte de las líneas que se desarrollan en los diferentes Institutos tendrán cabida en el nuevo Plan Nacional, ya que se incluirán buena parte de las dirigidas a estudios de procesos litosféricos (ausentes en el anterior Plan Nacional). Sin embargo, en el V Programa Marco, hay numerosas líneas que quedarán fuera, ya que preferentemente se incluyen aquellas que hacen referencia a medio ambiente y su desarrollo sostenible.

\section{Potencial científico}

\section{Institutos integrantes}

El número de personas por instituto es muy desigual, con un rango que va desde centros en el que participa un único científico del CSIC (Instituto Botánico de Barcelona) hasta el que cuenta con 42 (Museo Nacional de Ciencias Naturales).

\section{Institutos propios del Area}

CEAB. Centro de Estudios Avanzados de Blanes.

CIDE. Centro de Investigaciones sobre Desertificación.

EBD. Estación Biológica de Doñana

EEZA. Estación Experimental de Zonas Aridas.

IACT. Instituto Andaluz de Ciencias de la Tierra.

IAG. Instituto de Astronomía y Geodesia.

IATS. Instituto de Acuicultura «Torre de la Sal».

IBB. Instituto Botánico de Barcelona. 
ICM. Instituto de Ciencias del Mar.

ICMA. Instituto de Ciencias Marinas de Andalucia.

ICTJA. Instituto de Ciencias de la Tierra «Jaume Almera».

IGE. Instituto de Geología Económica.

IPE. Instituto Pirenaico de Ecología.

IREC. Instituto de Investigaciones en Recursos Cinegéticos.

MNCN. Museo Nacional de Ciencias Naturales.

RJB. Real Jardín Botánico

Institutos compartidos con otras Areas

CCMA. Centro de Ciencias Medioambientales.

CEBAS. Centro de Edafología y Biología Aplicada del Segura.

EEZ. Estación Experimental del Zaidin.

IARN. Instituto de Agrobiotecnología y Recursos Naturales de Navarra.

IBMB. Instituto de Biología Molecular de Barcelona.

IIM. Instituto de Investigaciones Marinas.

IMEDEA. Instituto Mediterráneo de Estudios Avanzados.

IPNA. Instituto de Productos Naturales y Agrobiología.

IRNAS. Instituto de Recursos Naturales y Agrobiología de Sevilla.

IRNASA. Instituto de Recursos Naturales y Agrobiología de Salamanca.

Unidades Asociadas y Laboratorios Europeos Asociados

1. Geociencias Marinas: Depto. Geología, Dinámica Geofísica y Palentología (Univ. Barcelona) - CSIC (ICM).

2. Atapuerca: Dept. Paleontología (Univ. Complutense de Madrid)

- Dept. Ciencias de la Tierra (Univ. Zaragoza) - Dept. Geografía e Historia (Univ. Rovira i Virgili de Tarragona)- CSIC (MNCN).

3. Laboratorio de Petrología Aplicada. Univ. de Alicante - Instituto de Geología Económica.

4. Grupo de Estado Sólido. Univ. de Salamanca - IRNASA.

5. Grupo de Hidrología Subterránea. Univ. Politécnica de Cataluña - CSIC (ICTJA).

6. Laboratorio Europeo Asociado de Ciencias del Mar.

Institutos implicados: CSIC: (ICM. CEAB). Universidad de Barcelona.Universidad de Perpignan. Laboratoire Arago. Banyuls-sur-Mer.

7. Unidad de Contaminación Atmosférica: CSIC (ICTJA, IQA), CIEMAT, Centro de Estudios Mediambientales del Mediterráneo. 


\section{Personal}

Nota: datos hasta 1998 y teniendo en cuenta solamente los Centros considerados en la Memoria del CSIC como adscritos al Area.

\begin{tabular}{|l|r|}
\hline \multicolumn{1}{|c|}{ Personal } & Número \\
\hline Profesores de Investigación & 28 \\
Investigadores & 62 \\
Investigador Titular & 144 \\
Personal Universitario & 148 \\
Doctores vinculados & 6 \\
Doctores contratados (MEC+CSIC) & 69 \\
Becarios & 246 \\
Otro personal contratado & 330 \\
Titulados & 86 \\
Ayudantes y Auxiliares & 142 \\
Administrativos & 54 \\
Personal Laboral & 164 \\
Subalternos y otros & 15 \\
\hline
\end{tabular}

\section{Líneas de investigación}

La actividad científica de los grupos de investigación es de buen nivel como lo avala el número de publicaciones y los proyectos (nacionales e internacionales) que desarrollan. Ello implica una correcta adecuación de los investigadores a las líneas prioritarias marcadas por la CICYT y DGICYT y por la Unión Europea. El Area temática de Recursos Naturales es, por su naturaleza, compleja y pluridisciplinar abarcando una amplia gama de disciplinas. Las principales líneas desarrolladas por los Institutos del CSIC son las siguientes:

\section{Geoquímica y Procesos superficiales}

Geoquímica teórica, Geotermómetros, Paleoclimatología, Geoquímica ambiental: Estudio de los elementos terrestres, explicando su distribución y formación, así como sus estructuras cristalinas. Análisis de la evolución del clima en la Tierra que permitan discernir la influencia antrópica. Estudio de la naturaleza y abundancia de los elementos constitutivos de la Tierra. Conocimiento de los orígenes de los cambios climáticos. Efecto real de las emisiones de origen antrópico Estudio de los procesos que controlan la distribución de los elementos en las rocas y su posterior dispersión y concentración en el suelo y agua. 


\section{Estructura y génesis minerales}

Mineralogía experimental y ambiental, Metalogenia, Biomineralización: Formación de minerales y procesos de cristalización. Línea relacionada con el Area de Ciencias de Materiales. Determinación precisa de las condiciones termodinámicas de formación de los minerales. Efecto de los minerales en la salud. Estudio de la composición y condiciones físicas de los fluidos mineralizantes, y de la fuente de los metales y la edad de la mineralización y de las rocas encajantes. Búsqueda de nuevos materiales, análisis del crecimiento cristalino.

Evolución y dinámica de la litosfera

Componentes mantélicos e Interacción astenosfera-litosfera. Formación de orógenos y cuencas sedimentarias. Volcanismo. Tectónica en tiempo real: Procesos en las capas superficiales de la corteza terrestre. Dinámica terrestre, geoquímica, litosfera, geofísica, sísmica, tectónica, petrología, sedimentología y paleontología. Estudio de la dinámica de los volcanes y sus consecuencias en analizar las repercusiones tanto sociales como científicas. Estudio de la deformación de la litosfera, tanto en movimientos rápidos (terremotos) o lentos (levantamiento o subsidencia).

\section{Ciencias del Suelo}

Procesos en Suelos, Hidrología: Edafología, erosión, desertificación, planificación territorial, degradación y conservación. Estudio del ciclo del agua, usos y optimización de los recursos. Aguas subterráneas, circulación, evolución. Conocimiento de los procesos que se producen e los suelos y su efecto en la agricultura y la degradación de los suelos.

\section{Estructura y procesos oceánicos}

Modelos de circulación, Balance de materia y energía: Oceanografía física y química, dinámica oceánica. Flujos biogeoquímicos, oceanografía, producción, influencia en la dinámica oceánica y clima.

\section{Sistemas litorales}

Fenómenos físicos y de transporte, Flujos biogeoquímicos, Dinámica de poblaciones de organismos: Estudio de los procesos que tienen lugar 


\section{Recursos Naturales}

en nuestro litoral, donde se concentra la mayor parte de la población y donde revierte buena parte de sus actividades de origen industrial y lúdica. Circulación, oceanografía, contaminación, playas, costas.

Balance elemental en la zona litoral y su papel como modulador del intercambio entre tierra y mar. Estudio de proliferación de especies nocivas o invasoras.

\section{Acuicultura y Pesquerías}

Reproducción y mejora genética, Nutrición y regulación del crecimiento, Patología de especies cultivadas, Biología y dinámica de recursos renovables: Optimización de los procesos de producción en acuicultura y explotación pesquera. Conocimiento amplio de procesos fisiológicos. Biología y dinámica de especies sometidas a explotación y consecuencias de dicha actividad en los ecosistemas. Acuicultura, genética, reproducción. Estudio de los mecanismos reproductores en especies de interés comercial y conocimiento de las posibilidades de manipulación y mejora para su cultivo en cautividad. Patologías infecciosas en los sistemas extensivos e intensivos de cultivo. Estudio de la biología de los organismos, su dinámica, así como del efecto de la pesca en los ecosistemas.

\section{Ecología evolutiva y del comportamiento}

Evolución de estrategias vitales, Comportamiento y estructura social, Evolución de sistemas reproductivos, Relaciones planta-animal, Ecología química: Conocimiento de la biología y ecología de especies de interés comercial o necesarias de especial protección y de sus ecosistemas. Estudio de la influencia de los factores ecológicos sobre los patrones y sistemas reproductivos, reparto de recursos, dispersión, etc. Análisis observacional y experimental de la influencia de factores ecológicos sobre los distintos elementos que configuran el comportamiento animal. Procesos de polinización y dispersión de semillas. Lucha biológica, farmacología, interacciones químicas entre especies.

\section{Ecología de poblaciones}

Demografía de especies, Ecología funcional: Estudio del funcionamiento de nuestros ecosistemas, con especial referencia a las áreas protegidas. Poblaciones, ecología, demografía, biología de la conservación. Producción biológica, Ecofisiología, biomarcadores. 
Flora y Fauna ibéricas

Fauna y Flora, Paleontología: Estudios de biodiversidad, necesidad de conocer qué especies existen en nuestro entorno y su evolución Fósiles, prehistoria, evolución.

\section{Futuro de la investigación sobre Recursos Naturales en el CSIC}

El Area de Recursos es por definición captadora de técnicas o resultados desarrollados en otras Areas. Es un Area que debe «endurecerse» en algunas de sus líneas, especialmente en las que son más descriptivas, con el fin de hacerlas más finalistas y sólidas. Algunos de los problemas, por ejemplo medio ambientales, podrían solucionarse de mejor manera si existiera una mejor coordinación entre especialistas de diferentes campos. En este sentido la interacción con el Area de Químicas (por ej. para estudios de biomarcadores, ecología química) o de Biología (ej. genética de poblaciones) y Materiales (ej. biocristales) sería deseable. Asimismo, la interacción con grupos de Física Teórica en temas relacionados con la dinámica de las poblaciones podría ser positiva. En líneas generales, la estructura de la I+D en el Area presenta algunos puntos frágiles en medios materiales y de personal, por lo que el reto para los próximos años es la consolidación de la situación actual y el mantenimiento del desarrollo creciente que ha tenido lugar en los últimos años.

Existe una escasa tradición en los Institutos y grupos del Area en interactuar entre sí, con las consiguientes desventajas a la hora de conseguir determinados proyectos y, en especial, en los europeos. $\mathrm{El}$ ejemplo de las investigaciones en el Guadiamar, a raíz del desastre originado por la rotura de la presa en Aznalcóllar, es emblemático y ha supuesto la creación de grupos pluridisciplinares, que actualmente han pedido proyectos FEDER y europeos. Es evidente que la estructura de los proyectos nacionales y la forma de financiar a los grupos ha condicionado, al menos en parte, la atomización. Sin embargo, debería favorecerse la creación de estos grupos, que seguro obtendrán sus beneficios en proyectos europeos e industriales. Debe recalcarse que, aunque alcanzar una masa crítica en cada Instituto es deseable, la verdadera masa crítica debe buscarse en la cooperación entre los grupos de los distintos Institutos del CSIC. Es ahí donde está nuestro mayor potencial y es en este campo interdisciplinar donde avanzará nuestra ciencia. 


\section{Recursos Naturales}

La investigación sobre los recursos naturales es actualmente una prioridad en todos los países de nuestro entorno. Un buen ejemplo lo constituyen programas internacionales en los que nuestro país está y cuya presencia y actividad debe potenciarse: Programa Mundial de Investigación del Clima, el Programa Internacional Biosfera-Geosfera, el Programa de Medio Ambiente y Desarrollo Sostenible del V Programa Marco de la Unión Europea. Por otra parte, existen numerosos Acuerdos y Convenciones internacionales relacionados con el medio ambiente, y que implican iniciativas para su mejor conocimiento, conservación, funcionamiento, gestión y aprovechamiento sostenible entre las que cabe destacar la Convención para la Diversidad Biológica, la Convención para la Lucha contra la Desertificación. Sin duda el conocimiento de los procesos que tienen lugar en cada componente del sistema es imprescindible para una correcta gestión de nuestros recursos. La adquisición de un nivel adecuado de conocimiento sobre el funcionamiento de nuestro entorno, permitirá a España sustentar las políticas nacionales e internacionales de gestión y conservación de los recursos adecuadas.

España es uno de los países de la Unión Europea con mayores recursos geológicos, biológicos y climáticos, a los que hay que unir su relación con el paisaje, usos turísticos y de ocio, entre otros. Un mejor conocimiento de los recursos y una optimización de su gestión repercute en prácticamente cualquier actividad económica. Los intereses económicos de España en actividades relacionadas con los ecosistemas marinos y terrestres son muy importantes. Un porcentaje significativo del PIB dependen directa o indirectamente de la explotación y uso de tales ecosistemas, siendo el sector turístico (mayoritariamente costero) el más significativo. Existen, además, en España recursos de diversa naturaleza y elevada demanda, sirva como ejemplo, los yacimientos minerales y de rocas ornamentales cuyos productos son deficitarios en muchos de los países de la UE.

Las principales dificultades del Area se centran en la ausencia de buenas y eficaces infraestructuras para investigación y en el número relativamente escaso de investigadores. A las carencias ya mencionadas de infraestructuras, habría que sumar una gran deficiencia en equipos de adquisición de datos. En los próximos años deberían acometerse algunas deficiencias y dificultades detectadas en años anteriores. Por un lado, como ya se ha señalado anteriormente, la falta de masa crítica en los grupos de investigación se traduce en una reducida capacidad de respuesta a los problemas de investigación y desarrollo y limita la participación en proyectos multidisciplinares y la transferencia de 
resultados. De hecho, en España, la relación entre número de investigadores estables y número de habitantes o entre número de investigadores e indicadores económicos como el PIB es muy inferior a la de otros países europeos. Asimismo, el crecimiento de años anteriores ha sesgado la composición de la comunidad de investigadores, dejando algunas disciplinas poco consolidadas. Entre ellas se podrían destacar las siguientes: Procesos en suelos, Geoquímica, Ecología Vegetal, Limnología, Ecología química, Fisiología animal, Química ambiental, Biomineralización, Genética de poblaciones, Oceanografía física y química, Biología de la conservación.

Considerando las disciplinas que históricamente se han desarrollado en el Area, así como los objetivos que se plantean en los Planes de investigación nacionales y europeos, los grandes objetivos que el Area debería considerar serían, entre otros, los siguientes aspectos:

1. Las investigaciones deberán incluir aquellos aspectos que permitan una optimización de las técnicas de vigilancia de la meteorología medioambiental aplicada a situaciones de riesgo de contaminación urbana y de los restantes ecosistemas, así como el estudio sobre la vulnerabilidad de ecosistemas a cambios climáticos. Ello implica mejorar la capacidad de observación con el fin de establecer indicadores del estado de contaminación de la atmósfera y la caracterización de la variabilidad climática y desarrollo de la capacidad de predicción climática.

2. Deberán potenciarse aquellos aspectos que permitan un mejor conocimiento del suelo y del subsuelo, así como de los procesos que en ellos actúan para comprender su capacidad de generar o almacenar recursos, permitir una óptima preservación, explotación y utilización de los mismos. Deberán incentivarse estudios integrados de modelización geológica del territorio incluyendo los recursos geológicos susceptibles de generar riqueza, la protección del medio natural frente su explotación y otras actuaciones antrópicas y la prevención y mitigación de los riesgos geológicos. Las necesidades de la sociedad requieren la potenciación de líneas aún poco desarrolladas en nuestro país dirigidas hacia conservación y gestión integral de los recursos hídricos, degradación y erosión del suelo, efectos de los cambios de uso del suelo y procesos de desertificación. Deberán promoverse estudios que permitan, asimismo, mejorar el conocimiento de los procesos básicos relacionados con los recursos hídricos superficiales y subterráneos, la calidad de sus aguas y factores de contaminación, procesos de salinización y su efecto en los suelos, permitiendo la estimación de caudales ecológicos y una adecuada ordenación del territorio. 


\section{Recursos Naturales}

3. El interés creciente por establecer las bases de un desarrollo sostenible de nuestro entorno implica el estudio integral del uso de los recursos naturales y ambientales, que permitan la introducción de técnicas innovadoras para la conservación y restauración de los recursos. Debe entenderse que el objetivo principal es la conservación y el uso sostenible de los recursos vivos, lo que requiere el conocimiento de su biología, factores que determinan la distribución, dinámica y abundancia de las especies y de aquellos procesos ligados a las formas de explotación o que afectan a su conservación. Asimismo, deben considerarse con especial atención proyectos dirigidos a la catalogación de las especies de flora y fauna silvestres, especialmente aquellos que incorporen técnicas moleculares para la identificación de especies y poblaciones.

4. Los resultados obtenidos en anteriores programas de investigación han detectado la complejidad de los problemas relacionados con los recursos marinos y las respuestas necesarias deben estar supeditadas a un adecuado conocimiento científico. Las investigaciones en este campo deben llevarse a cabo con una aproximación multidisciplinar que requiere en muchos casos el uso de infraestructuras de investigación importantes (ej. buques oceanográficos). Los esfuerzos deberán encaminarse hacia estudios que evalúen las consecuencias, a diferentes escalas espaciales, de los cambios naturales e inducidos por las actividades de origen antrópico en los océanos y las tendencias a corto, medio largo plazo en los ecosistemas. Asimismo, profundizar en un mejor conocimiento del funcionamiento de los ecosistemas marinos y el desarrollo de estudios integrados, especialmente en la franja costera, donde la influencia humana es más ostensible.

5. Dada la incidencia que las actividades humanas tienen sobre nuestros ecosistemas, incluidos los urbanos, deberán contemplarse estudios ambientales pluridisciplinares que incluyan la detección y evaluación de los agentes contaminantes y su efecto sobre las especies y ecosistemas. Ello implica la estrecha interacción con líneas de investigación desarrolladas en otras Areas científicas (por ejemplo, química ambiental, biomedicina) donde se realizan estudios que tendrían una interesante aplicación en problemas ambientales. El Area de Recursos Naturales, por la naturaleza de las investigaciones que desarrolla, debe incentivar este tipo de proyectos pluridisciplinares, que permitirán una mejor comprensión de los procesos que tienen lugar en nuestro entorno. 